REVIEW

\title{
Fusidic acid resistance in Staphylococcus aureus
}

\author{
D Dobie, J Gray
}

Arch Dis Child 2004;89:74-77. doi: 10.1136/adc.2003.019695

This review summarises current knowledge of the microbiological and clinical aspects of fusidic acid resistance in Staphylococcus aureus, and makes recommendations about fusidic acid prescribing and further research.

See end of article for authors' affiliations

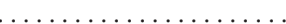

Correspondence to: Dr J Gray, Department of Microbiology, Birmingham Children's Hospital, Steelhouse Lane, Birmingham B4 6NH, UK; jim.gray@bch.nhs.uk

Accepted 21 June 2003
F usidic acid is a narrow spectrum antibiotic derived from Fusidium coccineum that has been used for over 35 years to treat infections with Staphylococcus aureus. ${ }^{1}$ Fusidic acid resistance in $S$ aureus can be readily selected for by in vitro exposure to the antibiotic, leading to the recommendation that for systemic therapy fusidic acid should only be given in combination with another agent. More controversial is the use of topical fusidic acid in the treatment of cutaneous and soft tissue infections. Many authors have suggested that the drug is effective as monotherapy in this setting. In the UK usage has of topical fusidic acid (alone or in combination with a glucocorticoid) almost doubled between 1995 and 2001,2 which has coincided with reports of rapidly increasing fusidic acid resistance from many centres. ${ }^{2-8}$

Increasing fusidic acid resistance in $S$ aureus might be important for three reasons. First, it might mean that systemic fusidic acid can no longer be used in situations where it is clinically indicated. Second, failure of topical treatment may be occurring, especially in primary care where treatment is often empiric, and third resistance to fusidic acid might be linked to other antibiotic resistances, therefore favouring spread of multiply antibiotic resistant $S$ aureus such as MRSA (methicillin resistant $S$ aureus).

\section{MODE OF ACTION OF FUSIDIC ACID}

Elongation factor $\mathrm{G}$ (EF-G) is a bacterial protein that is necessary for translocation on the bacterial ribosome after peptide bond formation during protein synthesis. Fusidic acid binds to this protein and the ribosome thereby inhibiting further bacterial protein synthesis. Prokaryotes possess only one type of elongation factor Eukaryotes, however, have other factors that are unaffected by the action of fusidic acid, which allow them to continue the process of protein synthesis. ${ }^{9}$ The action of fusidic acid is largely bacteriostatic, but at high concentrations (2 to 32-fold higher than the MIC) the effect may be bactericidal.

\section{ANTIBACTERIAL SPECTRUM OF ACTION AND CLINICAL USES}

Fusidic acid has an unusual spectrum of activity that includes corynebacteria, nocardia, anaerobes, and Neisseria species, but is used almost exclusively as an anti-staphylococcal agent. ${ }^{9}$ Its main indications are:

- Systemic treatment for severe staphylococcal infections. Oral or intravenous fusidic acid may be used in combination with another antistaphylococcal agent for the treatment of severe staphylococcal infections. ${ }^{10}$ It is particularly valuable for the treatment of osteomyelitis and septic arthritis, because of its excellent bone penetration. ${ }^{11}$

- Systemic treatment for colonisation and infection with MRSA. Fusidic acid has proven useful when combined with agents such as vancomycin, but not as monotherapy, in the treatment of infections with MRSA. ${ }^{12}$ Combined with rifampicin, it is used for attempted clearance of MRSA colonisation where topical agents such as mupirocin have failed. ${ }^{13}$

- Topical treatment of skin infections. Topical formulations of fusidic acid, now make up two thirds of the total usage ${ }^{14}$ and are widely used as monotherapy for the treatment of superficial cutaneous infections, including impetigo, folliculitis, erythrasma, furunculosis, and infected traumatic wounds. ${ }^{15}{ }^{16}$ Unfortunately, good evidence of efficacy is lacking as few studies are randomised, double blinded, or controlled. ${ }^{15}$

- Topical treatment of atopic dermatitis. Topical preparations of fusidic acid and a glucocorticoid are widely used in the treatment of atopic dermatitis. Skin colonisation with $S$ aureus is a characteristic feature of atopic dermatitis, and is believed to drive the inflammatory process, leading to the recommendation that fusidic acid-glucocorticoid preparations may be the treatment of choice for this condition. ${ }^{17}$

\section{RESISTANCE TO FUSIDIC ACID}

In vitro, resistance to fusidic acid can readily be selected from an initial high inoculum. ${ }^{18}$ However, there has been surprisingly little detailed study of fusidic acid resistance mechanisms in $S$ aureus.

All bacterial populations produce spontaneous single step chromosomal mutations in the gene coding for EF-G at a rate of 1 in $10^{6}-10^{8}$ cell divisions. This occurs even with isolates from individuals who have never been exposed to fusidic acid. It has been postulated that resistant mutants are slower growing and less virulent, but it is not clear if this is true. ${ }^{18}$ Resistance may also arise from plasmid mediated decreased cell wall or membrane permeability. ${ }^{18} 19$ This resistance may be linked to resistance to heavy metals and to other antibiotic resistances, including 
pencillinase production. ${ }^{19}$ The relative importance of these two resistance mechanisms is uncertain. Lacey and Rosdahl ${ }^{20}$ determined the resistance mechanism in 26 patient isolates, and found plasmid mediated resistance in 18. Resistance in the other strains was assumed to be chromosomal. Various other resistance mechanisms have been reported, but are less well characterised. ${ }^{18}$

TRENDS IN FUSIDIC ACID RESISTANCE IN S AUREUS There were a considerable number of reports of fusidic acid resistance rates in S aureus during the 1960s-1980s, which are summarised by Turnbridge and Collignon. ${ }^{18}$ However, until recently there has been comparatively little contemporary data. Two types of data have been widely reported. Fusidic acid resistance rates in blood culture isolates relate to the potential application of systemic fusidic acid for deep seated staphylococcal infections, whereas data relating to isolates from skin and soft tissue sites pertain to possible indications for topical therapy.

Studies of $S$ aureus bacteraemia up to the mid-1980s consistently showed fusidic acid resistance rates of $0-2.3 \%$, with no significant difference between hospital and community acquired cases. ${ }^{21-23}$ However, data from the UK suggest that there has subsequently been a rapid increase in the prevalence of fusidic acid resistance in blood culture isolates of $S$ aureus. The rate of fusidic acid resistance in methicillin susceptible $S$ aureus reported through the voluntary PHLS reporting scheme increased from $2.0 \%$ to $6.4 \%$ between 1990 and 2001. ${ }^{14}$ MRSA were excluded from this analysis in order to eliminate the possible influence of clonal dissemination of MRSA. In our hospital, between 1995 and 2002, 18/322 $(5.6 \%)$ blood culture isolates of $S$ aureus were fusidic acid resistant ( $\mathrm{J}$ Gray, unpublished data). The fusidic acid resistance rate in methicillin sensitive strains was $7.3 \%$, whereas no MRSA were fusidic acid resistant. The increasing rate of fusidic acid resistance in blood culture isolates of $S$ aureus is therefore not directly linked to the increase in MRSA bacteraemia that has occurred in the UK since the mid-1990s. ${ }^{24}$

Studies of fusidic acid resistance in isolates of $S$ aureus from skin and soft tissue sites have shown much more variation in fusidic acid susceptibility, with resistance rates of $26-33 \%$ in burns, dermatology, and ITU patients. ${ }^{18}$ Although in a recent Dutch study no fusidic acid resistance was detected in $S$ aureus isolated from children presenting to primary care with impetigo, ${ }^{25}$ in the UK fusidic acid resistance rates of between $11.5 \%$ and $18.5 \%$ have recently been reported, ${ }^{2-8}$ with especially high rates in children. ${ }^{35}$ While fusidic acid resistant strains are likely to be over-represented in these observational studies (because swabs from therapy resistant cases are more likely to be sent to the laboratory), resistance rates in laboratory isolates of $S$ aureus have nevertheless increased by $50-200 \%$ during the $1990 \mathrm{~s}^{2-8}$ A recent audit in our own hospital showed that 30 of $44(68 \%)$ isolates of $S$ aureus from children presenting to the Dermatology Department with impetigo were fusidic acid resistant. Even $50 \%$ of isolates from children with no history of fusidic acid treatment were fusidic acid resistant (SJ Beswick, unpublished data). Even more strikingly, in another study, where only two of the 46 children had received prior fusidic acid treatment, the resistance rate was $37.0 \%$.

\section{FUSIDIC ACID PRESCRIBING AND THE EMERGENCE OF RESISTANCE}

The ease with which fusidic acid resistance can be selected for in vitro is well established, and has led to the widely held view that fusidic acid as monotherapy risks treatment failure through selection of resistant mutants. ${ }^{1{ }^{18}}$ However, a limited number of studies, often involving relatively small numbers of patients, suggests that short term systemic or topical treatment for acute infections is clinically and microbiologically effective. For acute skin and soft tissue infections, 5-10 day courses of fusidic acid have been associated with a low incidence $(0-3.9 \%)$ of fusidic acid resistance emerging at the end of treatment. ${ }^{18}$ However, these studies have rarely examined the impact of treatment on $S$ aureus simultaneously present at carriage sites other than the site of infection. In one recent study, short term therapy was not associated with an increased fusidic acid resistance rate in post-treatment skin and nasal swabs. ${ }^{26}$ However, only a small number of patients were studied, of whom $26 \%$ already had fusidic acid resistant $S$ aureus in pretreatment swabs. Where treatment has been less successful is in hospitalised patients with chronic dermatological conditions, burns, or leg ulcers, where fusidic acid resistance rates of up to $43 \%$ following treatment have been reported. ${ }^{27}$ This may be because the higher bacterial burden in patients with underlying skin disease and/or extensive infection may increase the risk of selection of resistant mutants; because microbiological cure is rarely achieved in such infections, again favouring the selection of resistant mutants; or because patient to patient transmission of fusidic acid resistant strains is occurring, favoured by the underlying skin condition and the selective pressure of fusidic acid use. Whatever the reason, hospital outbreaks can be successfully controlled by restricting the use of topical fusidic acid, coupled with improved infection control precautions. ${ }^{28} 29$

On the basis that short courses of fusidic acid as monotherapy have been reported to be effective, and that until recently most reports of high rates of fusidic acid resistance are in hospitals, it has been suggested that it is appropriate to use fusidic acid in primary care. A recent randomised trial in the Netherlands perpetuated this view, by concluding that fusidic acid as monotherapy was the treatment of choice for impetigo in children. ${ }^{25}$ However, what the study actually showed was that topical fusidic acid administered together with povidone iodine was superior to povidone iodine alone: povidone iodine would have reduced the risk of selection of fusidic acid resistant mutants.

Recent evidence from the UK casts doubt on the view that fusidic acid use outside hospitals is justified. Although it must be borne in mind that samples are sent to the laboratory from only a small fraction of $S$ aureus infections in primary care, there is evidence that the rate of fusidic acid resistance is increasing at least as rapidly in the community as in hospitals, and that this increase parallels increased prescribing of topical fusidic acid..$^{2-8}$ Moreover, a direct association between resistance rates and fusidic acid prescribing in different Welsh general practices has recently been reported. ${ }^{30}$ Although fusidic acid resistance outside hospitals might be considered to be more likely to be due to selection of resistant mutants, rather than clonal dissemination, there are reports from the $\mathrm{UK}^{3}$ and Sweden ${ }^{31}$ of person to person transmission of fusidic acid resistant $S$ aureus in the community.

The prevalence of MRSA in dermatology patients is increasing, raising concern that widespread use of topical fusidic acid may be a contributory factor. ${ }^{32}{ }^{33}$ In the UK, the predominant epidemic strains of MRSA have remained fusidic acid sensitive. However, a new epidemic strain (EMRSA-17) that is fusidic acid resistant has recently been identified in a number of centres, especially in southern England..$^{34}$ Thus while there is no direct evidence that fusidic acid use to date has promoted spread of epidemic strains of MRSA, it may become more important in the future.

\section{RECOMMENDATIONS FOR THE USE OF FUSIDIC ACID IN TREATMENT OF INFECTIONS WITH $S$ AUREUS}

Topical antimicrobials are better tolerated than oral medication, which may improve compliance, particularly in 
children. ${ }^{35}$ For acute superficial skin infections, the efficacy of topical fusidic acid or mupirocin has been shown to be at least comparable to oral treatment, other than in more severe cases of impetigo. ${ }^{36-40}$ A small number of studies comparing topical fusidic acid and mupirocin in staphylococcal skin infections have shown little difference in clinical outcome, ${ }^{41} 42$ although in one study mupirocin was more effective in impetigo. ${ }^{43}$

Because of the perceived risk of promoting emergence and spread of resistance with topical antibiotic therapy, it is recommended that antibiotics used topically should be ones that are not used systemically. ${ }^{44}$ While mupirocin fulfils this criterion, it is important also to recognise that mupirocin is the cornerstone of MRSA eradication therapy, and that widespread use risks affecting the ability of hospitals to control the spread of MRSA. ${ }^{13}$

Clinical Evidence, the UK Department of Health/BMJ information resource, concluded that there is no evidence to support the general use of antibiotic therapy in atopic dermatitis, ${ }^{45}$ a view that has subsequently been endorsed by other authors. ${ }^{46}{ }^{47}$ Topical antibiotics are best avoided in any chronic skin condition, because of both doubtful efficacy and the higher risk of resistance emerging during therapy, at least with fusidic acid. ${ }^{27-29}$ For acute superficial infections, the convenience of topical antibiotic therapy needs to be balanced against the risks of promoting clinically important resistance. Neither fusidic acid nor mupirocin are ideal from this point of view, especially in hospitals, and either agent must be used with caution. There are few data on alternative agents.

The use of systemic fusidic acid is much less controversial in that, where it is clinically indicated, it is already used almost exclusively as combination therapy.

\section{CONCLUSION}

In the UK at least, we are witnessing a rapid increase in the fusidic acid resistance rate in both the community and hospitals. Although this has happened at the same time as the incidence of MRSA has increased, the two observations are not directly related. The increase in resistance threatens to render fusidic acid ineffective for clinically important indications, such as the treatment of deep seated infections and the control of MRSA. Resistance can also lead to failure of topical therapy in primary care, which may in turn be leading to children presenting to hospital with worsening infections.

We have only a limited understanding of fusidic acid resistance at the genetic, epidemiological, and clinical level, and too many of our prescribing practices are based on outmoded data obtained from observational studies of small numbers of patients. There is a lack of good quality studies examining the clinical efficacy of topical fusidic acid in skin and soft tissue infections. We do not understand the impact of fusidic acid prescribing in the community on the emergence and spread of resistance, and whether any duration of monotherapy is safe. We do not know the relative importance of the different mechanisms of fusidic acid resistance, or the extent to which clonal spread in the community is contributing to the increase in fusidic acid resistance. Further studies are required to address questions such as these. In the meantime, given the paucity of suitable alternatives to fusidic acid for topical administration, it is difficult to recommend completely outlawing its use. However, we believe that it is sensible to restrict use of topical fusidic acid to short courses for patients outside hospital without underlying skin conditions, together with close monitoring of local antibiotic susceptibility patterns. Systemic fusidic acid should continue to be used only in combination with other agents where clinically indicated, and where the infecting bacteria are susceptible.

\section{ACKNOWLEDGEMENTS}

The work of Dr SJ Beswick in collecting antibiotic histories for patients presenting with impetigo at Birmingham Children's Hospital is acknowledged.

\section{Authors' affiliations}

D Dobie, J Gray, Department of Microbiology, Birmingham Children's Hospital, Steelhouse Lane, Birmingham B4 6NH, UK

\section{REFERENCES}

1 Verbist L. The antimicrobial activity of fusidic acid. J Antimicrob Chemother 1990;25(suppl B): 1-5.

2 Brown EM, Wise R. Fusidic acid cream for impetigo. Fusidic acid should be used with restraint. BMJ 2002;324:1394.

3 Owen SE, Cheesbrough JS. Fusidic acid cream for impetigo. Findings cannot be extrapolated. BMJ 2002;324:1394.

4 Zadik P, Young N. Fusidic acid cream for impetigo. Resistance trends must be monitored. BMJ 2002;324:1394.

5 Stoddart B, Collyns T, Denton M. Fusidic acid cream for impetigo. Problem may be clinically important. BMJ 2002;324:1394.

6 Weston VC, Boswell TC, Finch RG, et al. Fusidic acid cream for impetigo. Emergence of resistance to fusidic acid limits its use. BMJ 2002;324:1394.

7 Sule O, Brown N, Brown DF, et al. Fusidic acid cream for impetigo. Judicious use is advisable. BMJ 2002;324:1394.

8 Brown EM, Thomas P. Fusidic acid resistance in Staphylococcus aureus isolates. Lancet 2002;359:803.

9 Collignon P, Turnidge J. Fusidic acid in vitro activity. Int J Antimicrob Agents 1999;12(suppl 2):S45-58.

10 Eykyn SJ. Staphylococcal bacteraemia and endocarditis and fusidic acid. $J$ Antimicrob Chemother 1990;25(suppl B):33-8.

11 Coombs RHH. Fusidic acid in staphylococcal bone and joint infection. $J$ Antimicrob Chemother 1990;25(suppl B):53-60.

12 Whitby M. Fusidic acid in the treatment of methicillin-resistant Staphylococcus aureus. Int J Antimicrob Agents 1999:12(suppl 2):S67-71.

13 Working Party Report. Revised guidelines for the control of methicillinresistant Staphylococcus aureus infection in hospitals. J Hosp Infect 1998;39:259-90.

14 Livermore D, James D, Duckworth G, et al. Fusidic acid use and resistance. Lancet 2002;360:806

15 Spelman D. Fusidic acid in skin and soft tissue infections. Int J Antimicrob Agents 1999;12(suppl 2):S59-66.

16 Wilkinson JD. Fusidic acid in dermatology. Br J Dermatol 1998;139:37-40.

17 Abeck D, Mempel M. Staphylococcus aureus colonisation in atopic dermatitis and its therapeutic implications. Br J Dermatol 1998;139:13-16.

18 Turnidge J. Collignon P. Resistance to fusidic acid. Int J Antimicrob Agents 1999; 12(suppl 2):S35-44

19 O'Brien FG, Price C, Grubb WB, et al. Genetic characterisation of the fusidic acid and cadmium resistance determinants of staphylococcus aureus plasmid pUB101. J Antimicrob Chemother 2002;50:313-21.

20 Lacey RW, Rosdahl VT. An unusual 'penicillinase plasmid' in Staphylococcus aureus; evidence for its transfer under natural conditions. J Med Microbiol 1974;7:1-9.

21 Bruun B, Sloth K, Weis Bentzon M, et al. A study of bacteraemias in Denmark from 1977-1978. Acta Path Micobiol Immunol Scand 1982:90(sect B):309-17.

22 Ispahani P, Pearson NJ, Donald FE. Blood cultures: eight years' experience of a conventional in-house system and trends in antimicrobial susceptibilities. Med Lab Sci 1989;46:295-304.

23 Phillips I, King A, Gransden WR, et al. The antibiotic sensitivity of bacteria isolated from the blood of patients in St. Thomas' Hospital, 1969-1988. J Antimicrob Chemother 1990;25(suppl C):59-80.

24 Anon. Methicillin resistance in Staphylococcus aureus isolated from blood in England and Wales: 1994 to 1998. CDR Weekly 1999;9:65-8.

25 Koning S, van Suijlekom-Smit LWA, Nouwen JL, et al. Fusidic acid cream in the treatment of impetigo in general practice: double blind randomised placebo controlled trial. BMJ 2002;324:203-6.

26 Ravenscroft JC, Layton AM, Eady AM, et al. Short-term effects of topical fusidic acid or mupirocin on the prevalence of fusidic acid resistant (FusR) Staphylococus aureus in atopic eczema. Br J Dermatol 2003; 148:1010-17.

27 Ayliffe GAJ. Use of antibiotics and resistance. In: Geddes AM, Williams JD, eds. Current antibiotic therapy. London: Churchill Livingstone, 1973:53-63.

28 Ayliffe GA Green W, Livingston R, et al. Antibiotic-resistant Staphylococcus aureus in dermatology and burns wards. J Clin Pathol 1977;30:40-4.

29 Ayliffe GAJ, Lilly HA, Lowbury EJL. Decline of the hospital staphylococcus? Incidence of multiresistant Staph. aureus in three Birmingham hospitals. Lancet 1979;1:538-41.

30 Mason BW, Howard AJ, Magee JT. Fusidic acid resistance in community isolates of methicillin-susceptible Staphylococcus aureus and fusidic acid prescribing. J Antimicrob Chemother 2003;51:1033-6.

31 Osterlund A, Eden T, Olsson-Liljequiust B, et al. Swedish Study Group on Fusidic-Acid-resistant Staphylococcus aureus. Clonal spread among Swedish 
children of a Staphylococcus aureus strain resistant to fusidic acid. Scand J Infect Dis 2002;34:729-34.

32 Farrell AM, Shanson DC, Ross JS, et al. An outbreak of methicillin-resistant Staphylococcus aureus (MRSA) in a dermatology day-care unit. Clin Exp Dermatol 1998:23:249-53.

33 Trividic M, Gauthier ML, Sparsa A, et al. Methi-resistant Staphylococcus aureus in dermatological practice: origin, risk factors and outcome. Ann Dermatol Venereol 2002; 129:27-9.

34 Aucken HM, Ganner M, Murchan S, et al. A new UK strain of epidemic methicillin-resistant Staphylococcus aureus (EMRSA-17) resistant to multiple antibiotics. J Antimicrob Chemother 2002;50:171-5.

35 Eady EA, Cove JH. Topical antibiotic therapy: current status and future prospects. Drugs Exp Clin Res 1990;16:423-33.

36 Rice TD, Duggan AK, DeAngelis C. Cost-effectiveness of erythromycin versus mupirocin for the treatment of impetigo in children. Pediatrics 1992;89:210-14

37 McLinn S. A bacteriologically controlled, randomised study comparing the efficacy of $2 \%$ mupirocin ointment (Bactroban) with oral erythromycin in the treatment of patients with impetigo. J Am Acad Dermatol 1990;22:883-5.

38 Mertz PM, Marshall DA, Eaglstein WH, et al. Topical mupirocin treatment of impetigo is equal to oral erythromycin therapy. Arch Dermatol 1989; 125:1069-73.
39 Villiger JW, Robertson WD, Kanji K, et al. A comparison of the new topical antibiotic mupirocin ('Bactroban') with oral antibiotics in the treatment of skin infections in general practice. Curr Med Res Opin 1986;10:339-45.

40 Pakrooh H. A comparison of sodium fusidate ointment ('Fucidin') alone versus oral antibiotic therapy in soft-tissue infections. Curr Med Res Opin 197778;5:289-94.

41 Gilbert M. Topical $2 \%$ mupirocin versus $2 \%$ fusidic acid ointment in the treatment of primary and secondary skin infections. J Am Acad Dermatol 1989;20:1083-7.

42 Morley PA, Munot LD. A comparison of sodium fusidate ointment and mupirocin ointment in superficial skin sepsis. Curr Med Res Opin 1988;11:142-8.

43 White DG, Collins PO, Rowsell RB. Topical antibiotics in the treatment of superficial skin infections in general practice-a comparison of mupirocin with sodium fusidate. J Infect 1989;18:221-9.

44 Anon. Editorial. Antibiotic resistance and topical treatment. BMJ 1978;2:649-50.

45 Charman C. Clinical evidence. Atopic eczema. BMJ 1999:318:1600-4.

46 Raimer SS. Managing pediatric atopic dermatitis. Clin Pediatr (Phila) 2000;39:1-14

47 Williams RE. The antibacterial-corticosteroid combination. What is its role in atopic dermatitis? Am J Clin Dermatol 2000;1:211-15.

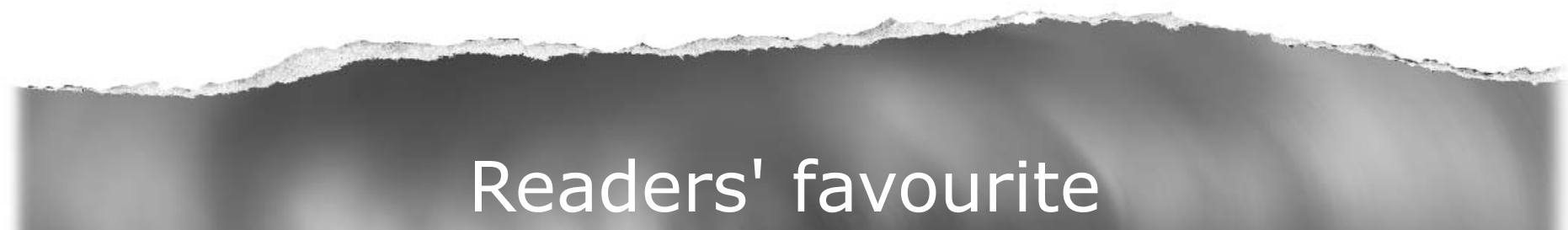

\section{Top 10}

Click on the "Top 10" button on the homepage to see which are the best read articles each month

\section{www.archdischild.com}

